PANORAMAS OF MATHEMATICS

BANACH CENTER PUBLICATIONS, VOLUME 34

INSTITUTE OF MATHEMATICS

POLISH ACADEMY OF SCIENCES WARSZAWA 1995

\title{
FROM THE THEOREM OF WAŻEWSKI TO COMPUTER ASSISTED PROOFS IN DYNAMICS
}

\author{
MARIAN MROZEK \\ Instytut Informatyki, Uniwersytet Jagielloński \\ Nawojki 11, 30-072 Kraków, Poland \\ E-mail:mrozek@ii.uj.edu.pl
}

1. Introduction. In 1947 Tadeusz Ważewski [35, 36] proved his famous Retract Theorem. Roughly speaking the theorem states that if the trajectories of a flow leave a closed set $W$ through a subset $W^{-}$of the boundary of $W$, do it "transversally", and the set $W^{-}$is not a deformation retract of $W$ then there exists a trajectory of the flow entirely contained in $W$. (We put the word transversally in quotation marks, because what really matters is a certain topological type of transversality.) The theorem soon found many applications in differential equations concerning the existence of bounded trajectories.

Twenty years later Charles C. Conley and his student Robert Easton started working on extending the concept of the Morse index to the case of a degenerate stationary point $[6,7]$. (Let us recall that the Morse index of a hyperbolic stationary point is the dimension of its unstable manifold.) The main tool they used was the concept of isolating block. Though originally they were not aware of the work of Ważewski, they soon realized that the notion of isolating block is closely related to the set $W$ in the assumption of the Retract Theorem. (Conley called such sets Ważewski sets.) Inspired by the proof of the Ważewski theorem Conley observed (comp. [4]) that the generalization of the Morse index proposed

1991 Mathematics Subject Classification: Primary: 34C35, 54H20. Secondary: 58F13, 58F22, $58 \mathrm{~F} 25,65 \mathrm{~L} 99,65 \mathrm{G} 10$.

Lecture given at the Banach Center Colloquium on 9th December 1993.

Research partially supported by KBN grant No 0449/P3/94/06.

The paper is in final form and no version of it will be published elsewhere. 
by him and R. Easton may be developed into an index theory for invariant sets. This lead to a large research project carried out under the direction of Conley by his Ph.D. students in 70's (comp. in particular $[3,22,16,17])$. The results of this research, scattered in several papers, were then nicely presented in a booklet [5] by Conley.

The basic concepts of the new theory, nowadays usually referred to as the Conley index theory, are the notions of an isolating neighbourhood and an isolated invariant set. A compact set $N$ is called an isolating neighbourhood if the maximal invariant subset of $N$ (possibly empty) does not intersect the boundary of $N$. In other words, $N$ is an isolating neighbourhood, if the trajectory of every point on the boundary of $N$ leaves $N$ in some positive or negative time (not necessarily immediately). An isolated invariant set $S$ is a compact invariant set which is a maximal invariant subset of some isolating neighbourhood $N$. The neighbourhood $N$ is then said to isolate $S$. It is easy to show that every Ważewski set is an isolating neighbourhood. It is much less straightforward to prove that every isolating neighbourhood $N$ isolating $S$ admits a subset $W$ which is a Ważewski set still isolating $S$. This done, one proves that the homotopy type (and in particular also the cohomology) of the quotient space $W / W^{-}$does not depend on the particular choice of the Ważewski set but only on the isolated invariant set $S$ inside. Thus, this homotopy type is an invariant of $S$ and is by definition the homotopy Conley index of $S$. The cohomology Conley index is defined as the cohomology of the homotopy Conley index.

Some people feel discouraged by the fact that the definition of the Conley index is quite complicated; in particular the index is not a number. Fortunately, though a formal axiomatization of the theory has not been performed yet, a set of properties, which seem to be sufficient in most applications, may be proposed. Let us also mention that several numbers may be extracted easily from this abstract form of the index. In particular, the Conley index of a hyperbolic stationary point is the homotopy type of an $n$-dimensional sphere with $n$ being the Morse index of that point.

The Retract Theorem of Ważewski rephrased in terms of the Conley index states that whenever the Conley index of an isolating neighbourhood is non-trivial (i.e. different from the homotopy type of the one point space) then it isolates a non-empty invariant set. One of the basic differences between the Retract Theorem and the Conley index consists in the fact that the index persists under a small perturbation of the flow. The computation of the Conley index is facilitated by several theorems, especially the homotopy property which states that the Conley index of an isolating neighbourhood $N$ remains constant when the flow is continuously changed but $N$ is kept an isolating neighbourhood. This gives the theory a flavour similar to the fixed point index theory, which does not contradict the fact that the two theories are essentially different. 
The potential strength of the Conley index theory lies in the fact that, unlike the Ważewski Theorem, the theory may be used not only to prove the existence of non-empty invariant sets but to tell something about their internal structure: the existence of stationary points, heteroclinic connections, periodic trajectories and recently also chaotic trajectories. This is possible, because the Conley indexes, as algebraic structures, may be somehow measured or compared. The results of measurements and comparisons often reflect the internal structure of an isolated invariant set.

One could then expect that the Conley index is a commonly used tool in differential equations but it is not. The Retract Theorem seems to be already applied everywhere it could have been applied. It is not surprising: the theorem is already 46 years old. As we said, the ambition of the Conley index theory is to find applications beyond the Ważewski Theorem. Unfortunately, it is even not easy to find isolating neighbourhoods unless a Ważewski set is known. The situation with Ważewski sets resembles that with Lyapunov functions: it is easy to check if something is a Lyapunov function but usually it is hard to find one. Paradoxically, numerical experiments indicate that the isolating neighbourhood is a very common phenomenon in dynamics. Thus, the behaviour of isolating neighbourhoods is opposite to Ważewski sets and Lyapunov functions: it is easy to find a candidate but hard to prove that we really found an isolating neighbourhood: the analytic technicalities are too complicated.

A numerical test for an isolating neighbourhood is simple. We choose a possibly large set of points in the boundary of the candidate $N$ and follow their trajectories numerically forwards and backwards for some time. If they all eventually leave the set $N$ we may believe that $N$ is an isolating neighbourhood. It is thus tempting to ask the question: could such a computation be converted into a rigorous proof? If yes, could we also use computer for rigorous computing the Conley index?

It is relatively easy to give positive answer to the first question. Since we can rigorously estimate all the errors involved in the computation, we can also make claims about trajectories of individual points. When the set of points in the boundary is large enough we can then extend the argument to all points in the boundary using Lipschitz estimates.

The answer to the other question is also positive but it requires a substantial extension of the Conley index theory. Any dynamics implemented on computer by its very nature is discrete. Thus we need the Conley index for discrete dynamical systems. Conley asked for such an extension but the problem remained unsolved for ten years. The obstacle seemed to be serious. The classical Conley index theory was build on the idea of homotopies along trajectories of the flow, the idea taken from Ważewski. In the discrete case such homotopies do not make any sense. Nevertheless a solution was finally proposed by J. Robbin and D. Salamon in 1988 in [33] and independently also in [25] in 1990. The other problem is that 
what we really deal with on computer is multivalued dynamics. This is because of error estimates. Thus a Conley index for discrete multivalued dynamical systems is needed. Such a construction was proposed in [15].

With the above set of tools computer assisted rigorous computation of the Conley index became, at least theoretically, possible. The first actual computation concerned chaos in the Lorenz equations [20,21]. Some other results based on similar methods, concerning the Kuramoto-Shivashinsky equation are in progress.

In this paper we would like to review basic results which lead to applying the ideas of Ważewski, via the Conley index, to computer assisted proofs in dynamics.

2. The Ważewski Retract Theorem. Let us begin with recalling the classical Ważewski Retract Theorem, following the original papers of Ważewski [35, 36, 37]. Ważewski studies a non-autonomous differential equation

$$
x^{\prime}=V(t, x),(t, x) \in W \subset \mathbf{R} \times \mathbf{R}^{n}
$$

with continuous right hand side which guarantees the uniqueness of solutions. Let $x\left(t, t_{0}, x_{0}\right)$ denote the solution of the equation originating from $\left(t_{0}, x_{0}\right)$. Assume $U \subset W$ is open. Take $\left(t_{0}, x_{0}\right) \in U$. Then there are two possibilities. Either the positive trajectory of $\left(t_{0}, x_{0}\right)$ is contained entirely in $U$ or it exits $U$ at some exit point $C\left(t_{0}, x_{0}\right)=\left(t^{\#}, x^{\#}\right) \in \mathrm{bd}_{W} U$. Ważewski calls the exit point a strong exit point if there exists an $\epsilon>0$ such that $\left(t, x\left(t, t^{\#}, x^{\#}\right)\right) \notin \operatorname{cl} U$ for $t^{\#}<t<t^{\#}+\epsilon$. Denote by $U^{\#}$ the set of all exit points and by $U^{-}$the set of strong exit points. Obviously $U^{-} \subset U^{\#} \subset \operatorname{bd}_{W} U$.

Recall that if $A \subset B$ then $A$ is called a retract of $B$ if there exists a continuous function $r: B \rightarrow A$, called retraction, such that $r_{\mid A}=\mathrm{id}_{A}$. $A$ is called a deformation retract of $B$ if there exists a retraction $r: B \rightarrow A$ which is homotopic to $\operatorname{id}_{\mid B}$.

Theorem 2.1. (Ważewski Retract Theorem). Assume $U^{\#}=U^{-}$, i.e. every exit point is a strong exit point. Let $Z \subset U \cup U^{-}$be such that $Z \cap U^{-}$is a retract of $U^{-}$but not a retract of $Z$. Then there exists a point $\left(t_{0}, x_{0}\right) \in Z \cap U$ such that its positive trajectory is contained entirely in $U$.

The classical formulation of the theorem is slightly complicated, so let us make a few comments. In most applications $Z$ is taken to be just $U \cup U^{-}$. The formulation may be slightly simplified if the open set $U$ is replaced by a closed set $W$. The condition $U^{\#}=U^{-}$is then equivalent to assuming that the set

$$
W^{-}:=\{(s, x) \in W \mid \exists \epsilon>0: x(t, s, x) \notin W \text { for } s<t<s+\epsilon\}
$$

is closed. Following C. Conley, we will call such sets Ważewski sets. Note that the points in $W^{-}$correspond to strong exit points in the Ważewski terminology but they are usually called just exit points, because the exit points in the sense of Ważewski are no longer necessary. The theorem then becomes: 
THeOREM 2.2. If $W$ is a Ważewski set and $W^{-}$is not a deformation retract of $W$ then there exists $x \in W$ such the whole positive trajectory of $x$ is contained in $W$.

The idea of proof is very simple. Assume there is no such trajectory. Then for every point $x \in W$ the associated exit point $C(x) \in W^{-}$is well defined. The closedness of $W^{-}$implies that the transformation $C: W \rightarrow W^{-}$is continuous, thus evidently it is a retraction. Moreover, using the trajectories of the flow, one can easily build a homotopy joining $C$ with the identity map on $W$. Thus $W^{-}$ turns out to be a deformation retract of $W$, a contradiction. The proof of the original version of Ważewski theorem goes along exactly the same lines.

Though it is rather hard to prove non-existence of retraction just from definition, many examples, in particular the fact that an $n$-dimensional ball cannot be retracted to an $(n-1)$-dimensional sphere are well known. (This is easy to prove only in dimension one, because in that case topology is induced by order.) Basic tools which are used to measure the non-retractability are provided by the homotopy theory and the homology theory. The idea of Conley was to apply these theories to transform the Ważewski Theorem into an index theory.

3. The Conley index. First of all observe that Ważewski Theorem by its very nature is purely topological; hence the same proof applies when the differential equation is replaced by a flow $\pi: X \times \mathbf{R} \rightarrow X$ on a topological space $X$. Recall that a flow is a group $\pi=\left\{\pi_{t}\right\}_{t \in \mathbf{R}}$ of homeomorphisms of $X$ indexed by real numbers and such that the map $t \rightarrow \pi_{t}$ is a homomorphism of groups. (The solutions of (1) define a flow $\pi=\left\{\pi_{t}:\left(t_{0}, x_{0}\right) \rightarrow x\left(t-t_{0}, t_{0}, x_{0}\right)\right\}$ on $W$.)

The classical Conley index is defined for flows. However, unlike the Ważewski Theorem, the theory requires some compactness. In the simplest setting, adopted in this paper, flows on locally compact metric spaces are considered. Thus assume that $\pi$ is a flow on a locally compact metric space $X$. The maximal invariant subset of $N \subset X$ is defined by

$$
\operatorname{Inv} N:=\left\{x \in N \mid \forall t \in \mathbf{R} \pi_{t}(x) \in N\right\} .
$$

We say that $S$ is invariant if $S=\operatorname{Inv} S$ and that $S$ is isolated invariant if there exists a compact set $N$ such that $S=\operatorname{Inv} N$ and $S \subset \operatorname{int} N$. The set $N$ is then called an isolating neighbourhood.

The definition of the homotopy (cohomological) Conley index for flows rests on the following two theorems.

Theorem 3.1. Every isolating neighbourhood $N$ contains a Ważewski set $W$ such that Inv $N \subset W$.

Theorem 3.2. The homotopy type of the quotient space $W / W^{-}$(the AlexanderSpanier cohomology $\left.H^{*}\left(W, W^{-}\right)\right)$does not depend on the index pair but only on the isolated invariant set $S$. 
This common value is taken as the homotopy (cohomological) Conley index.

Though the homotopy index is more general and easier to introduce (all necessary definitions from homotopy theory are elementary), the cohomological Conley index is easier to apply, because the cohomology is easier to compute than the homotopy type. Thus we will restrict our attention to the cohomology Conley index in the sequel. The cohomology Conley index takes the form of a graded vector space (for simplicity we take cohomology with field coefficients).

The definition of the Conley index for semidynamical systems is similar (see [34]) but conceptually more complicated, so we omit the details.

4. The Conley index for maps. When a differential equation is investigated numerically, what really happens is that a discrete numerical scheme approximating the equation is iterated. Thus, if there is any hope of computing the Conley index on computer, first of all the index must be generalized to discrete dynamical systems. A discrete dynamical system on $X$ is a group $\pi=\left\{\pi_{t}\right\}_{t \in \mathbf{Z}}$ of homeomorphisms of $X$ like in the case of a flow but indexed by integers. Unlike the flow, a discrete dynamical system is generated by a single element, namely $\pi_{1}$ (or $\pi_{-1}$ ). Hence it is often identified with its generator. In other words, if $f: X \rightarrow X$ is a homeomorphism then we can think of $f$ as a discrete dynamical system $\pi_{f}=\left\{f^{n}\right\}_{n \in \mathbf{Z}}$ given by the iterates of $f$.

The question how to generalize the Conley index to the discrete case was raised already in Conley's booklet [5]. The notions of isolating neighbourhood and isolated invariant set can be carried over directly to the discrete case via the obvious change of $\mathbf{R}$ to $\mathbf{Z}$. However, the notion of Ważewski set does not make sense in the discrete case. This is because the trajectories may jump over the boundary. Thus it is necessary to extend the notion of exit set in such a way that it is not necessarily a subset of boundary. This is done via so called index pairs.

Definition 4.1. The pair $P=\left(P_{1}, P_{2}\right)$ of compact subsets of $N$ will be called an index pair of $S$ in $N$ iff the following three conditions are satisfied

$$
\begin{gathered}
x \in P_{i}, f(x) \in N \Rightarrow f(x) \in P_{i}, \quad i=1,2 \\
x \in P_{1}, f(x) \notin N \Rightarrow x \in P_{2} \\
\operatorname{Inv} N \subset \operatorname{int}\left(P_{1} \backslash P_{2}\right) .
\end{gathered}
$$

Like in the case of a flow (Theorem 3.1) one can prove

Theorem 4.2. Every isolating neighbourhood admits an index pair P.

However, there is no direct analogue of Theorem 3.2. The cohomology of index pair does depend on its choice. The proof fails, because the fundamental tool in the proof of Theorem 3.2, i.e. the homotopy build along trajectories of the flow, does not make any sense in the discrete case. In this respect entirely new ideas are needed. 
Assume $f: X \rightarrow X$ is a homeomorphism, $S$ is an isolated invariant set with respect to $\pi_{f}$ and $P=\left(P_{1}, P_{2}\right)$ is an index pair in $N$. It can be easily derived from the definition of the index pair that $f$ induces a map of pairs

$$
f_{P}:\left(P_{1}, P_{2}\right) \ni x \rightarrow f(x) \in\left(P_{1} \cup f\left(P_{2}\right), P_{2} \cup f\left(P_{2}\right)\right)
$$

and the inclusion

$$
i_{P}:\left(P_{1}, P_{2}\right) \ni x \rightarrow x \in\left(P_{1} \cup\left(P_{2}\right), P_{2} \cup f\left(P_{2}\right)\right) .
$$

induces an isomorphism in Alexander-Spanier cohomology.

Definition 4.3. The endomorphism

$$
H^{*}\left(f_{P}\right) \circ H^{*}\left(i_{P}\right)^{-1} \text { of } H^{*}(P),
$$

where $H^{*}$ denotes the Alexander-Spanier cohomology, will be called the index map associated with the index pair $P$ and denoted by $I_{P}$.

The index map contains information which is essential in the construction of the discrete Conley index. This information is not important in the continuous case, because it is then trivial in the sense that the index map associated with the time-one translation map of a flow is always the identity.

In order to make use of the extra information we need some definitions.

Denote by $\mathcal{E}$ the category of vector spaces. If $E, F \in \mathcal{E}$ then $\mathcal{E}(E, F)$ will stand for the set of all linear maps from $E$ to $F$ in $\mathcal{E}$.

The category $\mathcal{E} E$ of linear endomorphisms is defined as follows. The objects of the category $\mathcal{E} E$ are all pairs $(E, e)$, where $E \in \mathcal{E}$ and $e \in \mathcal{E}(E, E)$ is an endomorphism. The morphisms from $(E, e) \in \mathcal{E} E$ to $(F, f) \in \mathcal{E} E$ are all maps $\phi \in \mathcal{E}(E, F)$ such that the diagram

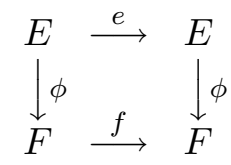

commutes. We define the category $\mathcal{E} I$ of linear automorphisms as the full subcategory of $\mathcal{E} E$ consisting of pairs $(E, e)$ such that $e$ is an isomorphism. We will consider the category $\mathcal{E}$ as a subcategory of $\mathcal{E} I$ using the natural functorial embedding

$$
\mathcal{E} \ni E \rightarrow(E, \text { id }) \in \mathcal{E} I \text {. }
$$

Thus we have

$$
\mathcal{E} \subset \mathcal{E} I \subset \mathcal{E} E .
$$

We want to define a functor $L: \mathcal{E} E \rightarrow \mathcal{E} I$. Since its definition is simplest on a subcategory of finite dimensional vector spaces, we restrict our attention to that case. 
Let $(F, f) \in \mathcal{E} E$. Define the generalized kernel of $f$ as

$$
\operatorname{gker}(f):=\bigcup\left\{f^{-n}(0) \mid n \in \mathbf{N}\right\} .
$$

Since $f(\operatorname{gker}(f)) \subset \operatorname{gker}(f)$, we have an induced monomorphism

$$
f^{\prime}: F / \operatorname{gker}(f) \ni[x] \rightarrow[f(x)] \in F / \operatorname{gker}(f) .
$$

Put

$$
L(F, f):=\left(F / \operatorname{gker}(f), f^{\prime}\right) .
$$

It is easy to verify that $f^{\prime}$ is in fact an isomorphism. Thus $L(F, f) \in \mathcal{E} I$. One can easily extend the above definition to morphisms, so that we actually obtain a covariant functor

$$
L: \mathcal{E} E \rightarrow \mathcal{E} I
$$

We will call it the Leray functor.

The analogue of Theorem 3.2 in the discrete case is the following theorem.

Theorem 4.4. Assume $f: X \rightarrow X$ is a homeomorphism and $S$ is an isolated invariant set with respect to $\pi_{f}$. Then $L\left(H^{*}(P), I_{P}\right)$ does not depend on the index pair but only on the isolated invariant set $S$.

The above theorem allows us to define the Conley index in the discrete case as $L\left(H^{*}(P), I_{P}\right)$. Let us emphasize that in the discrete case the Conley index has the form of a pair

$$
\mathrm{Con}^{*}(S)=\left(C H^{*}(S), \chi^{*}(S)\right),
$$

where $C H^{*}(S)$ is a graded vector space and $\chi^{*}(S): C H^{*}(S) \rightarrow C H^{*}(S)$ is a graded automorphism.

As we stated above, if $f$ is the time-one-map of a flow and $S$ is an isolated invariant set, then $I_{P}$ is an identity. Consequently

$$
\operatorname{Con}\left(S, \pi_{f}\right)=L\left(H^{*}(P), \mathrm{id}\right)=\left(H^{*}(P), \mathrm{id}\right)=H^{*}(P) .
$$

Hence we have the following

Theorem 4.5 (see [28]). The cohomological Conley index of an isolated invariant set of a flow coincides with the corresponding cohomological Conley index of the time-one-map of this flow.

There is also

THEOREM 4.6 (see [28]). $S$ is an isolated invariant set with respect to a flow iff it is an isolated invariant set with respect to the time-one translation of the flow.

Hence our index can be considered as a generalization of the cohomological Conley index for flows. 
5. Properties of the Conley index. As we mentioned in the introduction, the Conley index has several properties which facilitate its computation. In most applications it is sufficient to know the properties only. In this section we would like to summarize them.

The first property is just a reformulation of the Ważewski Retract Theorem.

THEOREM 5.1. Ważewski property: If $N$ is an isolating neighbourhood with respect to $\pi_{f}$ and $\operatorname{Con}\left(\operatorname{Inv} N, \pi_{f}\right) \neq 0$ then $\operatorname{Inv} N$ is non-empty.

TheOREM 5.2. Stability property: If $N$ is an isolating neighbourhood with respect to $\pi_{f}$ then it is an isolating neighbourhood with respect to $\pi_{g}$ for $g$ sufficiently close to $f$ in the compact-open topology and the Conley indexes with respect to $f$ and $g$ coincide.

TheOREM 5.3. Homotopy property: Assume $\pi_{s}, s \in[0,1]$, is a family of $d y$ namical systems on $X$ continuously depending on s. If $N \subset X$ is an isolating neighbourhood with respect to every dynamical system $\pi_{s}$ then $\operatorname{Con}\left(\operatorname{Inv} N, \pi_{s}\right)$ does not depend on $s$.

THEOREM 5.4. Additivity property: If $N, N_{1}, N_{2}$ are isolating neighbourhoods such that $\operatorname{Inv} N=\operatorname{Inv} N_{1} \cup \operatorname{Inv} N_{2}$, Inv $N_{1} \cap \operatorname{Inv} N_{2}=\emptyset$ then $\operatorname{Con}\left(\operatorname{Inv} N, \pi_{f}\right)=$ $\operatorname{Con}\left(\operatorname{Inv} N_{1}, \pi_{f}\right) \times \operatorname{Con}\left(\operatorname{Inv} N_{2}, \pi_{f}\right)$

Theorem 5.5. Normalization property: The whole space $X$ is an isolating neighbourhood and $\operatorname{Con}\left(X, \pi_{f}\right)=\left(H^{*}(X), f^{*}\right)$.

TheOREM 5.6. Commutativity property (see [29]). Assume $f=\psi \phi, g=\phi \psi$, where $\phi: X \rightarrow Y, \psi: Y \rightarrow X$ are continuous. If $S \subset X$ is an isolated invariant set with respect to $\pi_{f}$ then $\phi(S)$ is an isolated invariant set with respect to $\pi_{g}$ and $\operatorname{Con}\left(S, \pi_{f}\right)=\operatorname{Con}\left(\phi(S), \pi_{g}\right)$.

TheOREM 5.7. Relation to fixed point index (see [23]). Assume $X$ is a compact $A N R$ and $S$ is an isolated invariant set of $\pi_{f}$. Let $\operatorname{Con}\left(S, \pi_{f}\right)=(E, e) \in \mathcal{E} I$. Then $E$ is of finite type and $\Lambda(e)$, the Lefschetz number of $e$, is exactly the fixed point index of $f$ in a neighbourhood of $S$.

Let $x_{0}$ be a hyperbolic fixed point of $f$. Let $k$ denote the number of eigenvalues of $D f\left(x_{0}\right)$ with modulus greater than one (counted with multiplicity). Let $l$ denote the number of real eigenvalues of $D f\left(x_{0}\right)$ which are less than -1 . Then the pair $(k, l)$ will be called the Morse index of $x_{0}$.

TheOrem 5.8. Assume $x_{0}$ is a hyperbolic fixed point of a $C^{1}$-diffeomorphism $f: \mathbf{R}^{n} \rightarrow \mathbf{R}^{n}$. Then $\left\{x_{0}\right\}$ is an isolated invariant set and

$$
\operatorname{Con}_{i}\left(\left\{x_{0}\right\}, \pi_{f}\right)= \begin{cases}0 & \text { for } i \neq k \\ \left(\mathbf{Q},(-1)^{l} \mathrm{id}\right) & \text { for } i=k,\end{cases}
$$

where $(k, l)$ is the Morse index of $\left\{x_{0}\right\}$. 
6. The Conley index for multivalued discrete dynamical systems. In order to apply the Conley index in computer assisted proofs, it was necessary to extend the Conley index theory to multivalued maps. Such a theory for multivalued flows was proposed in [24]. The generalization in that case is quite natural. However, the discrete case is different, because it is not evident how to define an isolating neighbourhood in that case. In [15] an extension of the theory, based on the following definition of the isolating neighbourhood, was proposed.

Definition 6.1. Assume $\mathcal{F}: X \rightarrow X$ is a multivalued map and $N \subset X$. The set $\operatorname{Inv}(N, \mathcal{F})$ is defined as the set of $x \in N$ such that there exists a function $\sigma: \mathbf{Z} \rightarrow N$ satisfying $\sigma(0)=x$ and $\sigma(n+1) \in \mathcal{F}(\sigma(n))$. The set $N$ is said to be an isolating neighbourhood for $\mathcal{F}$ if

$$
B \operatorname{diam}_{N} \mathcal{F}(\operatorname{Inv} N) \subset \operatorname{int} N,
$$

where $\operatorname{diam}_{N} \mathcal{F}$ is the maximal diameter of the values of $\mathcal{F}$ in $N$.

The generalization of the notion of the index pair is then straightforward:

Definition 6.2. Let $N$ be an isolating neighbourhood for $F$. A pair $P=$ $\left(P_{1}, P_{2}\right)$ of compact subsets $P_{2} \subset P_{1} \subset N$ is called an index pair if the following conditions are satisfied:

$$
\begin{gathered}
\mathcal{F}\left(P_{i}\right) \cap N \subset P_{i}, i=1,2 ; \\
\mathcal{F}\left(P_{1} \backslash P_{2}\right) \subset N ; \\
\operatorname{Inv} N \subset \operatorname{int}\left(P_{1} \backslash P_{2}\right) .
\end{gathered}
$$

The definition of the index map does not essentially differ from the single valued case. One only needs to put some admissibility conditions (as in $[12,13]$ ) to ensure that the multivalued map induces a map on cohomology level. This is all what is necessary to develop the Conley index theory for multivalued discrete dynamical systems.

As we mentioned in the introduction, all what we may obtain rigorously from numerical computations of a trajectory of a differential equation is only a set where the exact value is located. This is why we need the Conley index theory for multivalued maps. The following result let us go back to the single-valued map of interest.

Theorem 6.3 (see [30]). Let $\mathcal{F}$ be an admissible multivalued map and $f$ be a selector of $\mathcal{F}$, i.e. $f(x) \in \mathcal{F}(x)$ for every $x \in \mathbf{R}^{n}$. If $N$ is an isolating neighbourhood for $\mathcal{F}$, then it is an isolating neighbourhood for $f$. Furthermore, the Conley indexes of $f$ and $\mathcal{F}$ coincide. 
7. Results in dynamics based on Conley index. We would like to show in this section that results based on the Conley index go essentially beyond the Ważewski Retract Theorem. Let us begin with an example from [5], where Conley index is used to prove the existence of bounded but non-stationary solutions.

EXAMPLE 7.1. Consider the system of differential equations

$$
\left\{\begin{array}{l}
x_{i}^{\prime}=x_{i+1} \\
x_{n}^{\prime}=\left(x_{1}\right)^{2}-1 .
\end{array} \quad i=1,2, \ldots, n-1\right.
$$

The system admits a non-stationary, bounded solution.

The sketch of the proof is as follows: Let $S$ denote the set of points which lie on bounded trajectories of system (1). It may be easily shown that $S$ is compact, i.e. it is an isolated invariant set. Moreover, system (1) can be continued to a system with no bounded trajectories. Thus the homotopy and Ważewski properties imply that $\operatorname{Con}(S)=0 . S$ contains the two stationary trajectories of (1), $S_{1}$ and $S_{2}$. It suffices to prove that $S \neq S_{1} \cup S_{2}$. Assume the contrary. Then, by the additivity property, $0=\operatorname{Con}(S)=\operatorname{Con}\left(S_{1}\right) \times \operatorname{Con}\left(S_{2}\right)$ and $\operatorname{Con}\left(S_{1}\right)=\operatorname{Con}\left(S_{2}\right)=0$. However, it can be computed that at least one of the stationary points $S_{1}, S_{2}$ must be hyperbolic and Theorem 5.8 implies that its index is non-zero, a contradiction.

Conley shows in [5] that system (2) is gradient-like in odd dimension. Thus the non-stationary solution is in fact a heteroclinic connection between the two stationary solutions. The Conley index turned out to be very useful in studying the existence of heteroclinic connections and a whole new theory, called the connection matrix theory, emerged from such applications (see $[9,10,11])$.

Consider now the following map obtained from (2) by the Euler approximation method

$$
f_{s}: \mathbf{R}^{n} \ni\left(x_{1}, x_{2}, \ldots x_{n}\right) \rightarrow\left(x_{1}, x_{2}, \ldots x_{n}\right)+s\left(x_{2}, \ldots, x_{n},\left(x_{1}\right)^{2}-1\right) \in \mathbf{R}^{n},
$$

where $s$ is a constant denoting the step of the Euler method. Similarly as in the continuous case one can show, using the discrete Conley index, that it also admits a non-constant, bounded trajectory at least for small values of the step $s$ (for a more general treatment see [32]).

Although until recently the problems of existence of heteroclinic connections as in the above example, were the main area of applications of the Conley index, the following two theorems substantially extend this area, by providing tools for proving the existence of periodic solutions and chaos.

Theorem 7.2 (Ch. McCord, K. Mischaikow and M. Mrozek, see [18]). Assume $X$ is an $A N R$ and $\varphi: X \times[0, \infty) \rightarrow X$ is a semiflow with compact attraction. If $N$ is an isolating neighbourhood for $\varphi$ which admits a cross-section $\Sigma$ and

$$
\operatorname{dim} C H^{2 n}(N, \varphi)=\operatorname{dim} C H^{2 n+1}(N, \varphi) \quad \text { for } n \in \mathbf{Z}
$$


or

$$
\operatorname{dim} C H^{2 n}(N, \varphi)=\operatorname{dim} C H^{2 n-1}(N, \varphi) \quad \text { for } n \in \mathbf{Z}
$$

and not all above dimensions are zero then $\varphi$ has a periodic trajectory in $N$.

Theorem 7.3 (K. Mischaikow and M. Mrozek, see [19]). Assume that $N=$ $N_{0} \cup N_{1}$ is an isolating neighbourhood under $f$ where $N_{0}$ and $N_{1}$ are disjoint compact sets and for $k=0,1$

$$
\operatorname{Con}^{n}\left(N_{k}\right)=\left\{\begin{array}{cl}
(\mathbf{Q}, \text { id }) & \text { if } n=1 \\
0 & \text { otherwise. }
\end{array}\right.
$$

Then $N_{k l}:=\left(N_{k} \cap f\left(N_{k}\right)\right) \cup\left(N_{k} \cap f\left(N_{l}\right)\right) \cup\left(N_{l} \cap f\left(N_{l}\right)\right)$ for $k, l \in\{0,1\}, k \neq l$, are isolating neighbourhoods and if additionally $\chi^{*}\left(N_{k l}\right)$ is not conjugate to identity then there exists a $d \in \mathbf{N}$ and a continuous surjection $\rho: \operatorname{Inv}(N, f) \rightarrow \Sigma_{2}$ such that

$$
\rho \circ f^{d}=\sigma \circ \rho
$$

where $\sigma: \Sigma_{2} \rightarrow \Sigma_{2}$ is the full shift dynamics on two symbols.

The above result allows to prove analytically chaos in the Hénon map (see $[20])$.

8. Computer assisted proofs in dynamics. Theorem 7.3 cannot be applied directly to a differential equation, because the automorphism part of the index is always identity in that case. However, the theorem could be applied to a Poincaré map. Unfortunately, in general there is no analytic formula for a Poincaré map, so in that case it is especially hard to find the necessary Conley indexes with analytic methods and a computer assisted argument seems to be a natural choice. Here is a rough idea of such a proof (for simplicity we assume that the problem is three dimensional). We select a Poincaré plain and a finite grid of squares on the plain covering the area of interest. Then we follow numerically the trajectories of the centres of the squares until they cross the Poincaré plain again. Let us call the map obtained this way the computed Poincaré map. We know it only approximates the exact Poincaré map to some extend. Having estimated all involved errors we obtain a multivalued map, defined at the centres of the grid. Its values are balls centered at the computed Poincaré map with radius being the error estimate. Thus the value of the exact Poincare map is contained in the ball. This multivalued map is not yet the one we need, because it is defined only at the finite set of the centres of the grid. Knowing a Lipschitz constant of the Poincaré map, we increase the radius of every ball in such a way that it contains not only the image of the centre of the square but the image of the whole square. Finally we assign the new ball as the value to all points in the corresponding square (the points at the boundaries of the squares require a bit more delicate treatment to obtain a well defined map; we omit the details here). The multivalued map obtained this way still has the 
property that the exact Poincaré map is its selector. We now verify (this is a combinatorial task again performed on computer) if the candidates for isolating neighbourhoods in Theorem 7.3 are isolating neighbourhoods for the computed multivalued map. If they are not, our proof fails. Otherwise we use Theorem 7.3 to carry over this fact to the exact Poincaré map and apply Theorem 7.3.

The discussed scheme was successfully applied to the Lorenz equations.

Theorem 8.1 (K. Mischaikow and M. Mrozek, see [20, 21]). Consider the Lorenz equations

$$
\begin{aligned}
\dot{x} & =s(y-x) \\
\dot{y} & =R x-y-x z \\
\dot{z} & =x y-q z,
\end{aligned}
$$

and the plane

$$
P:=\{(x, y, z) \mid z=53\} .
$$

For all parameter values in a sufficiently small neighbourhood of $(s, R, q)=$ $(45,54,10)$, there exists a Poincaré section $N \subset P$ such that the Poincaré map $g$ induced by (1) is Lipschitz and well defined. Furthermore, there exists a $d \in \mathbf{N}$ and a continuous surjection $\rho: \operatorname{Inv}(N, g) \rightarrow \Sigma_{2}$ such that

$$
\rho \circ g^{d}=\sigma \circ \rho
$$

where $\sigma: \Sigma_{2} \rightarrow \Sigma_{2}$ is the full shift dynamics on two symbols.

The situation when the presented scheme of computer assisted proofs fails requires some discussion. Though it may mean that the result we want to prove fails, more probably it just means that the size of squares in our grid was too large and our computations were not sufficiently accurate. Hence we can always decrease the size of squares and the accuracy of computations and repeat the procedure. This means more computations. Roughly speaking, if we want to increase accuracy $n$ times, we need $n^{d}$ more computations, with $d$ being the dimension of the problem. Thus it may turn out that the amount of computations necessary to get the positive result is far beyond the power of the best present days computers. This also explains why topological methods are better than smooth methods in this respect. If we need to compute a space derivative of a solution, an equation in variations must be simultaneously solved which immediately changes a 3-dimensional problem into a 12-dimensional problem.

The choice of Lorenz equations as a first candidate to a computer assisted proof was dictated (surprisingly) by the fact that the amount of computations necessary to complete the goal in that case was relatively small, though still quite complex. This also justified the particular choice of coefficients.

In fact, the main limitation of the method is the amount of necessary computations. However, the rapid progress in the available power of computers together 
with the development of more efficient algorithms gives us hope to attack more and more complex problems. The power of the method is the fact that it may provide rigorous results concerning concrete differential equations and dynamical systems which are intractable by todays analytic methods and are only investigated numerically. Because of the inherent nature of computational complexity in nonlinear problems, the method seems to be natural and maybe even inevitable.

Finally let us mention that the method offers a way of answering one of the fundamental questions of present days numerical analysis: how is the dynamics of the discretization of a differential equation related to the dynamics of the equation.

\section{References}

[1] O. Aberth, Precise Numerical Analysis, William C. Brown Publishers, Dubuque, Iowa, 1988.

[2] V. Benci, A Generalization of the Conley-Index Theory, Rend. Istit. Mat. Trieste 18 (1986), $16-39$.

[3] R. Churchill, Isolated invariant sets in compact metric spaces, J. Differential Equations 12 (1972), 330-352.

[4] C. Conley, On a generalization of the Morse index, in: Ordinary Differential Equations, 1971 NRL-MRC Conference, ed. L. Weiss, Academic Press, New York (1972), 27-33.

[5] C. C. Conley, Isolated invariant sets and the Morse index, CBMS no. 38, A.M.S., Providence, R.I., 1978.

[6] C. Conley, R. Easton, Isolated Invariant Sets and Isolating Blocks, in: Advances in Differential and Integral Equations, ed. J. Nohel, Studies in Applied Mathematics 5. SIAM Publications, Philadelphia (1969), 97-104.

[7] C. Conley, R. Easton, Isolated Invariant Sets and Isolating Blocks, Trans. Amer. Math. Soc. 158 (1971), 35-61.

[8] M. Degiovanni and M. Mrozek, The Conley index for maps in absence of compactness, Proc. Roy. Soc. Edinburgh Sect. A 123 (1993), 75-94.

[9] R. Franzosa, Index Filtrations and the Homology Index Braid for Partially Ordered Morse Decompositions, Trans. Amer. Math. Soc. 298 (1986), 193-213.

[10] R. Franzosa, The Connection Matrix Theory for Morse Decompositions, Trans. Amer. Math. Soc. 311 (1989) 561-592.

[11] R. Franzosa, The Continuation Theory for Morse Decompositions and Connection Matrices, Trans. Amer. Math. Soc. 310 (1988), 781-803.

[12] L. Górniewicz, Topological Degree of Morphisms and its Applications to Differential Inclusions, Raccolta di Seminari del Dipartimento di Matematica dell'Universita degli Studi della Calabria, No. 5, 1983.

[13] L. Górniewicz, Homological Methods in Fixed Point Theory of Multi-valued Maps, Dissertationes Math. 129, PWN, Warszawa, 1976. 
[14] A. Iserles, A. T. Peplow, A. M. Stuart, A unified approach to spurious solutions introduced by time discretization. Part I: Basic theory, SIAM J. Numer. Anal. 28 (1991), $1723-1751$.

[15] T. Kaczyński and M. Mrozek, Conley index for discrete multivalued dynamical systems, Topology Appl., accepted.

[16] H. L. Kurland, The Morse Index of an Isolated Invariant Set is a Connected Simple System, J. Differential Equations 42 (1981), 234-259.

[17] H. L. Kurland, Following Homology in Singularly Perturbed Systems, J. Differential Equations 62 (1986), 1-72.

[18] Ch. McCord, K. Mischaikow and M. Mrozek, Zeta Functions, Periodic Trajectories and the Conley Index, J. Differential Equations, accepted.

[19] K. Mischaikow and M. Mrozek, Isolating neighbourhoods and Chaos, Jap. J. Ind. \& Appl. Math., accepted.

[20] K. Mischaikow and M. Mrozek, Chaos in Lorenz equations: a computer assisted proof, Bull. Amer. Math. Soc., in print.

[21] K. Mischaikow and M. Mrozek, Chaos in Lorenz equations: a computer assisted proof, Part II: details, preprint.

[22] J. T. Montgomery, Cohomology of Isolated Invariant Sets under Perturbation, J. Differential Equations 13 (1973), 257-299.

[23] M. Mrozek, Index pairs and the Fixed Point Index for Semidynamical Systems with Discrete Time, Fund. Math. 133 (1989), 179-194.

[24] M. Mrozek, A Cohomological Index of Conley Type for Multi-valued Admissible Flows, J. Differential Equations 84 (1990), 15-51.

[25] M. Mrozek, Leray Functor and the Cohomological Conley Index for Discrete Dynamical Systems, Trans. Amer. Math. Soc. 318 (1990), 149-178.

[26] M. Mrozek, Open index pairs, the fixed point index and rationality of zeta functions, Ergodic Theory Dynamical Systems 10 (1990), 555-564.

[27] M. Mrozek, The Morse Equation in Conley's Index Theory for Homeomorphisms, Topology Appl. 38 (1991), 45-60.

[28] M. Mrozek, The Conley index on compact ANR's is of finite type, Results Math. 18 (1990), 306-313.

[29] M. Mrozek, Shape Index and Other Indices of Conley Type for Continuous Maps on Locally Compact Metric Spaces, Fund. Math., 145 (1994), 15-37.

[30] M. Mrozek, Topological invariants, multivalued maps and computer assisted proofs in dynamics, in preparation.

[31] M. Mrozek and K. P. Rybakowski, A cohomological Conley index for maps on metric spaces, J. Differential Equations 90.1 (1991), 143-171.

[32] M. Mrozek and K. P. Rybakowski, Discretized ordinary differential equations and the Conley index, J. Dynamics Differential Equations 4 (1992), 57-63.

[33] J. W. Robbin and D. Salamon, Dynamical systems, shape theory and the Conley index, Ergodic Theory Dynamical Systems 8 (1988), 375-393. 
[34] K. P. Rybakowski, The Homotopy Index and Partial Differential Equations, SpringerVerlag, Berlin Heidelberg 1987.

[35] T. Ważewski, Une méthode topologique de l'examen du phénomène asymptotique relativement aux équations différentielles ordinaires, Rend. Accad. Nazionale dei Lincei, Cl. Sci. fisiche, mat. e naturali, Ser. VIII, vol. III (1947), 210-215.

[36] T. Ważewski, Sur un principe topologique pour l'examen de l'allure asymptotique des intégrales des équations différentielles ordinaires, Ann. Soc. Polon. Math. 20 (1947), 279313.

[37] T. Ważewski, Sur un méthode topologique de l'examen de l'allure asymptotique des intégrales des équations différentielles, Proceedings of the International Congress of Mathematicians 1954, 3 (1955), 5-14.

[38] H. C. Yee, P. K. Sweby and D. F. Griffiths, Dynamical Approach Study of Spurious Steady-State Numerical Solutions of Nonlinear Differential Equations. 1. The Dynamics of Time Discretization and Its Implications for Algorithm Development in Computational Fluid Dynamics, J. Comput. Phys. 97 (1991), 249-310. 